\title{
BMJ Open Cost-effectiveness of HPV-based cervical cancer screening in the public health system in Nicaragua
}

\author{
Nicole G Campos, ${ }^{1}$ Mercy Mvundura, ${ }^{2}$ Jose Jeronimo, ${ }^{3}$ Francesca Holme, ${ }^{3}$ \\ Elisabeth Vodicka, ${ }^{4}$ Jane $\mathrm{J} \mathrm{Kim}^{1}$
}

To cite: Campos NG, Mvundura M, Jeronimo J, et al. Cost-effectiveness of HPV-based cervical cancer screening in the public health system in Nicaragua. BMJ Open 2017;7:e015048. doi:10.1136/ bmjopen-2016-015048

- Prepublication history and additional material are available. To view, please visit the journal (http://dx.doi.org/10.1136/ bmjopen-2016-015048)

Received 4 November 2016 Revised 9 March 2017 Accepted 30 March 2017

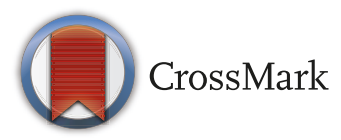

${ }^{1}$ Center for Health Decision Science, Harvard T.H. Chan School of Public Health, Boston, Massachusetts, USA

${ }^{2}$ PATH, Devices and Tools Program, Seattle, Washington, USA

${ }^{3}$ PATH, Reproductive Health Global Program, Seattle, Washington, USA

${ }^{4}$ University of Washington, School of Pharmacy, Seattle, Washington, USA

Correspondence to

Dr Nicole G Campos

ncampos@hsph.harvard.edu

\section{ABSTRACT}

Objectives To evaluate the cost-effectiveness of human papillomavirus (HPV) DNA testing (versus Papanicolaou (Pap)based screening) for cervical cancer screening in Nicaragua. Design A previously developed Monte Carlo simulation model of the natural history of HPV infection and cervical cancer was calibrated to epidemiological data from Nicaragua. Cost data inputs were derived using a microcosting approach in Carazo, Chontales and Chinandega departments; test performance data were from a demonstration project in Masaya department.

Setting Nicaragua's public health sector facilities.

Participants Women aged 30-59 years.

Interventions Screening strategies included (1) Pap testing every 3 years, with referral to colposcopy for women with an atypical squamous cells of undetermined significance or worse result ('Pap'); (2) HPV testing every 5 years, with referral to cryotherapy for HPV-positive eligible women (HPV cryotherapy or 'HPV-Cryo'); (3) HPV testing every 5 years, with referral to triage with visual inspection with acetic acid (VIA) for HPV-positive women ('HPV-VIA'); and (4) HPV testing every 5 years, with referral to Pap testing for HPV-positive women ('HPV-Pap').

Outcome measures Reduction in lifetime risk of cancer and incremental cost-effectiveness ratios (ICER; 2015 US\$ per year of life saved (YLS)).

Results HPV-based screening strategies were more effective than Pap testing. HPV-Cryo was the least costly and most effective strategy, reducing lifetime cancer risk by 29.5\% and outperforming HPV-VIA, HPV-Pap and Pap only, which reduced cancer risk by $19.4 \%, 12.2 \%$ and $10.8 \%$, respectively. With an ICER of US $\$ 320 / Y L S$, HPV-Cryo every 5 years would be very cost-effective using a threshold based on Nicaragua's per capita gross domestic product of US\$2090. Findings were robust across sensitivity analyses on test performance, coverage, compliance and cost parameters.

Conclusions HPV testing is very cost-effective compared with Pap testing in Nicaragua, due to higher test sensitivity and the relatively lower number of visits required. Increasing compliance with recommended follow-up will further improve the health benefits and value for public health dollars.

\section{BACKGROUND}

Cervical cancer is the leading cause of cancer among women in Nicaragua, with an
Strengths and limitations of this study

- Strengths of this study include the use of implementation data from the Scale-Up project to estimate the cost-effectiveness of human papillomavirus testing in Nicaragua's public health system. Findings were robust across extensive sensitivity and scenario analyses.

- A limitation of this study is that modelled screening algorithms do not entirely reflect the complex downstream management described by in-country guidelines. While the modelled algorithms reflect the prototypical structure of a screening episode and the type of facility at which visits usually take place, these do not capture variation due to geography or health facility capacity.

- An additional limitation is that while we adopted a micro-costing approach to leverage data from implementation in Nicaragua, individual-level data for each woman were not available; furthermore, we did not have cost data associated with HPV selfcollection in community settings, where most selfcollection takes place.

estimated 934 cases and 424 deaths each year. ${ }^{1}$ Yet, cervical cancer is preventable through screening that allows for early detection and subsequent treatment of precancerous lesions caused by sexually transmitted infection with human papillomavirus (HPV). While most HPV infections clear spontaneously within 1 to 2 years, a persistent infection with one of approximately 15 oncogenic HPV genotypes may progress to precancer which, if untreated, may become invasive cancer. ${ }^{23}$

In most high-income countries, routine screening with cervical cytology (ie, Papanicolaou (Pap)) testing has substantially reduced the incidence of cervical cancer. ${ }^{4}$ However, due to its low sensitivity to detect precancer, Pap testing must be performed at regular frequent intervals in women of screening age. In low-income and middle-income settings, where many women do not have access to routine primary healthcare 
and even fewer have access to higher-level facilities that offer diagnostic testing and treatment, Pap testing has not been effective at reducing cervical cancer incidence and mortality. ${ }^{5}$ In Nicaragua, an estimated $31.5 \%$ of women aged 15 to 49 years have been screened within the last year, and nearly $30 \%$ of women in this age group have never been screened. ${ }^{6}$ One recent survey found that $87 \%$ of women in León, Nicaragua, were informed of their Pap results, but of those who were referred to follow-up, only $67 \%$ received further care. ${ }^{7}$

HPV DNA tests are highly sensitive to detect potentially oncogenic HPV infections and present an alternative to Pap-based screening. Because HPV-negative women are at very low risk for developing cervical cancer within the next 10 years, ${ }^{8}$ the interval between screenings can be extended to at least 5 years for this subset of women. ${ }^{9}$ An additional advantage of HPV testing is that samples can be collected by a provider or by the woman herself, reducing the burden on health workers and time women spend seeking care and potentially increasing screening uptake. ${ }^{10-13}$ Furthermore, a lower-cost HPV DNA test known as careHPV has been clinically validated ${ }^{14}{ }^{15}$ and is now commercialised. Given these potential benefits, the WHO recommends HPV testing for countries with sufficient resources. ${ }^{9}$

In 2011, the Screening Technologies to Advance Rapid Testing for Cervical Cancer Prevention-Utility and Program Planning (START-UP) project in Nicaragua's Masaya department demonstrated that screening with careHPV could be effectively implemented in public sector health facilities. ${ }^{14}$ The Ministry of Health of Nicaragua subsequently built on these initial efforts, incorporating HPV testing into public healthcare systems in three departments with technical assistance from PATH under the Scale-Up project. Adoption of HPV testing within Nicaragua's public healthcare system is taking place in three phases. ${ }^{16}$ In phase 1 , partner organisations worked with the Ministry of Health to prepare for introduction of HPV screening into public health facilities by developing screening and treatment algorithms, creating educational materials, organising training sessions for health workers and laboratory technicians and bolstering referral and treatment systems for follow-up of screen-positive women. Phase 2 piloted screening with $10000 \mathrm{HPV}$ tests in order to identify and address barriers to implementation. Phase 3 will expand coverage to over 50000 women within 1 year.

To inform decision makers considering the national adoption and scale up of HPV testing within Nicaragua's public health sector, this study aimed to (1) estimate the economic cost of cervical cancer screening with careHPV testing and (2) project the long-term health and economic impact and value (ie, cost-effectiveness) of careHPV testing in Nicaragua relative to existing Pap-based screening.
METHODS

\section{Analytic overview}

We used a micro-costing approach to measure and aggregate the cost of all resources used to provide cervical cancer screening at the level of the individual patient within the public health sector in Nicaragua. We considered direct medical costs (ie, medical resources required for the intervention), direct non-medical costs (ie, other resources consumed as part of the intervention, such as patient transportation costs) and patient time costs (ie, time spent travelling and waiting for or receiving care). These cost data were input into a previously developed Monte Carlo simulation model (programmed in $\mathrm{C}++$ ) of the natural history of HPV infection and cervical cancer that was calibrated to epidemiological data from Nicaragua. ${ }^{1718}$ We then used the model to project the lifetime health and economic outcomes associated with careHPV testing, using three different algorithms for the management of women who test HPV positive and Pap-based screening for women aged 30 to 59 years.

Model outcomes included the lifetime risk of cervical cancer, total lifetime costs per woman (in 2015 US\$) and life expectancy. Incremental cost-effectiveness ratios (ICER) were calculated by dividing the additional cost of a particular strategy by its additional health benefit, compared with the next most costly strategy. Dominated strategies (defined as more costly and either less effective or having a higher incremental cost-effectiveness ratio than more effective strategies) were eliminated. There is no universal criterion that defines a threshold cost-effectiveness ratio, below which an intervention is considered good value for money; we considered an intervention with an ICER less than Nicaragua's 2015 per capita gross domestic product (GDP) of US $\$ 2090$ to be 'very cost-effective', and an intervention with an ICER less than three times per capita GDP as 'cost-effective'. ${ }^{19}$ We followed guidelines for cost-effectiveness by adopting a societal perspective, including costs irrespective of the payer in order to capture the opportunity cost of resources used for the screening intervention. We discounted future costs and life-years at a rate of 3\% per year to account for time preferences (Supplementary Data). ${ }^{20} 21$

\section{Mathematical simulation model}

Descriptions of the natural history model of HPV infection and cervical carcinogenesis and model parameterisation process have been previously published, ${ }^{17} 18$ but we summarise model features here. Individual girls enter the model at age 9 years, prior to initiating sexual activity, and face monthly transitions between mutually exclusive health states that reflect disease progression, including type-specific HPV infection, grade of precancer (ie, cervical intraepithelial neoplasia (CIN) grade 2 or 3 ) and stage of invasive cancer. Transition probabilities may vary by age, HPV type, duration of infection or precancerous lesion status, prior HPV infection and exposure to screening and treatment of HPV or precancer. Cervical cancer can be detected through symptoms or screening. 


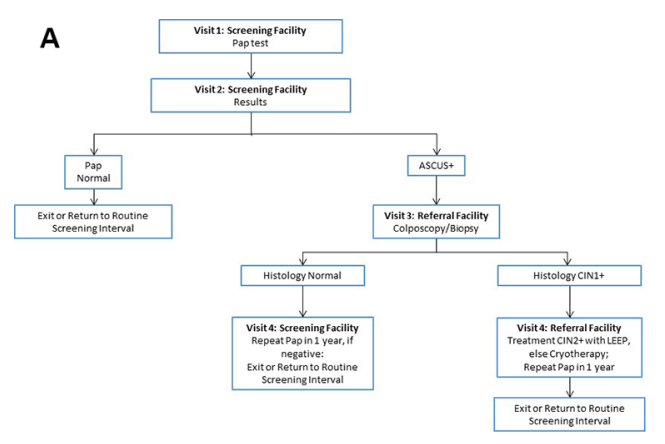

B
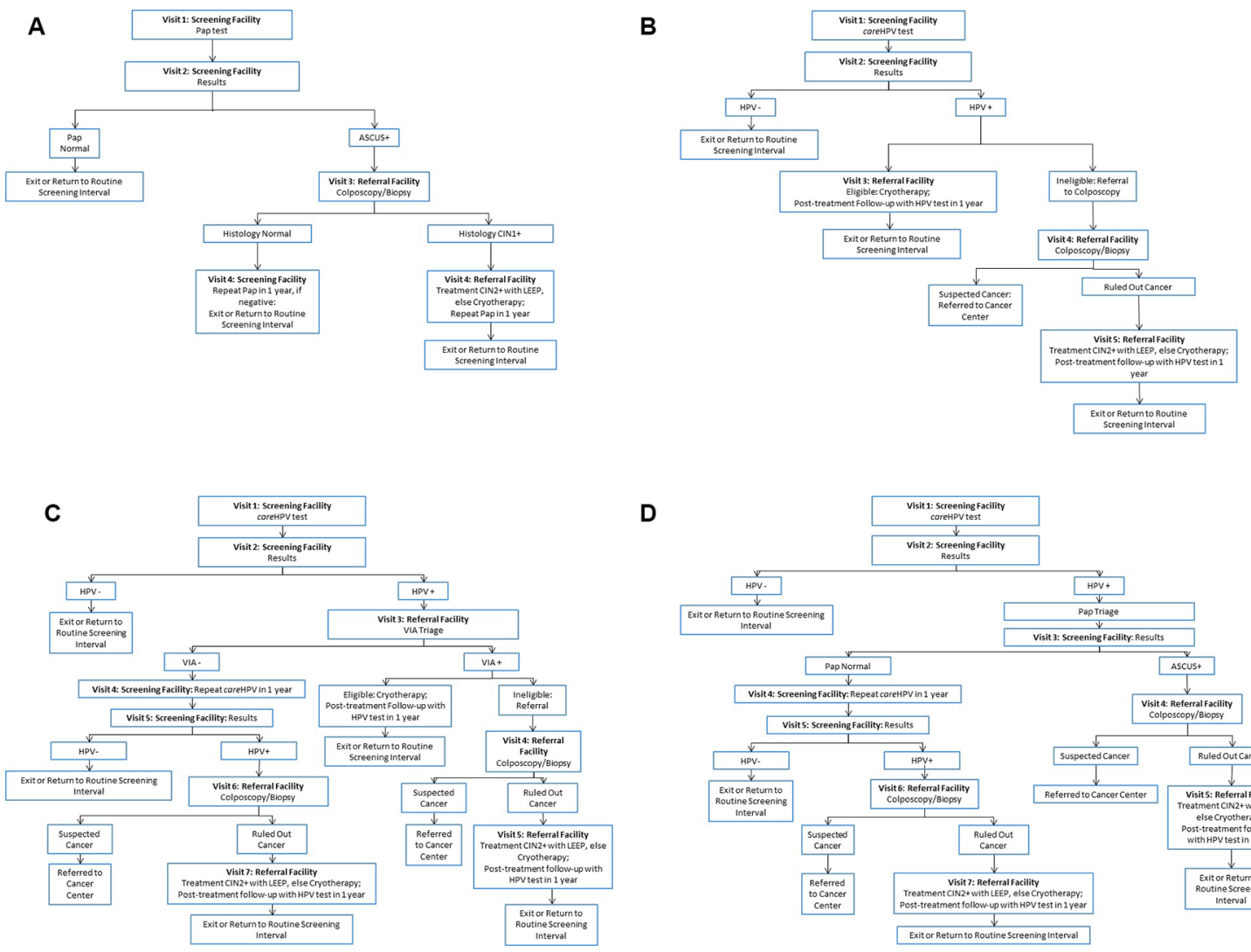

D

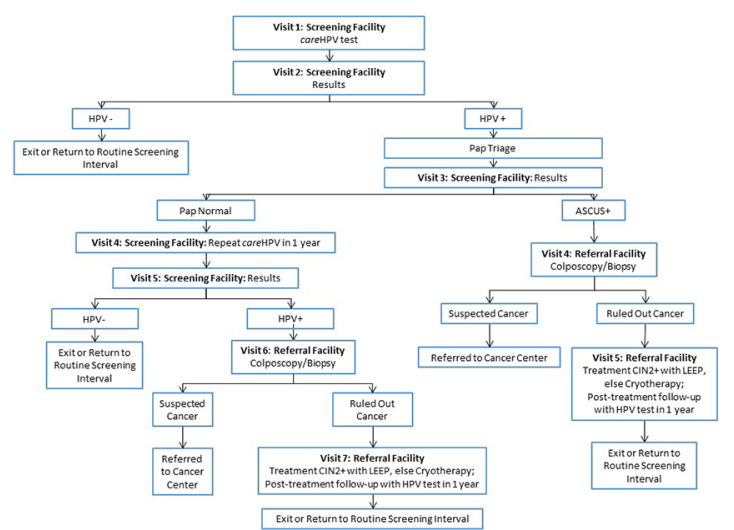

Figure 1 Pathways of care, by screening strategy. Each diagram indicates the flow of screening-eligible women (ie, women aged 30 to 59 years) through each point of contact in a screening episode, conditional on visit compliance and test results, for (A) Pap testing every 3 years (Pap), which requires four visits for screening, diagnosis and treatment; (B) HPV testing with referral to cryotherapy for all HPV-positive eligible women every 5 years (HPV-Cryo), which requires three or more visits for screening and necessary treatment; (C) HPV testing followed by visual inspection with acetic acid (VIA) triage of HPV-positive women every 5 years (HPV-VIA), which requires three or more visits for screening and necessary treatment; and (D) HPV testing followed by Pap triage of HPV-positive women every 5 years (HPV-Pap), which requires five or more visits for screening and necessary treatment. ASCUS, Pap result of atypical squamous cells of undetermined significance or worse; CIN, cervical intraepithelial neoplasia; HPV, human papillomavirus; LEEP, loop electrosurgical excision procedure; VIA, visual inspection with acetic acid.

Death can occur from non-cervical causes or from cervical cancer after its onset. The model tracks each individual woman's health status, clinical events and economic outcomes over her lifetime and aggregates outcomes to estimate the expected costs and health outcomes over the lifetime of the cohort.

The model was calibrated to epidemiological data on age-specific HPV prevalence and cervical cancer incidence from Nicaragua. ${ }^{1417}$ We estimated baseline 'prior' input parameter values for natural history transitions using available longitudinal data, including age- and type-specific HPV incidence data from Colombia. ${ }^{22-25}$ To reflect potential differences in parameters that may vary by setting (ie, age-specific and type-specific HPV incidence, natural immunity following initial infection) and uncertainty in progression and regression of precancer, we set plausible bounds around these input values and performed repeated model simulations of disease natural history in the absence of any intervention. Each model simulation selected one random value within the bounds for each uncertain parameter, creating a unique natural history input parameter set. By summing the log-likelihood of model-projected outcomes for each parameter set relative to the epidemiological data from Nicaragua, we computed a goodness-of-fit score. We selected the 50 top-fitting input parameter sets to use in analysis. Results are reported as the mean across the top 50 parameter sets, and ICER are reported as the ratio of the mean costs divided by the mean effects of one strategy versus another across sets. ${ }^{26}$ Further details on model parameterisation, including calibration, are available in the online supplementary appendix.

\section{Cervical cancer screening strategies}

We considered the following screening strategies for women aged 30 to 59 years (figure 1): (1) Pap testing every 3 years, with referral to colposcopy for all women with an atypical squamous cells of undetermined significance or worse result (hereafter referred to as 'Pap'); (2) careHPV testing every 5 years, with referral to cryotherapy for all HPV-positive eligible women (HPV cryotherapy or 'HPV-Cryo'); (3) careHPV testing every 5 years, with referral to triage with visual inspection with acetic acid (VIA) for all HPV-positive women ('HPV-VIA'); and (4) 
careHPV testing every 5 years, with referral to Pap testing for all HPV-positive women ('HPV-Pap'). The pathway of care for each strategy was based on patterns of care in the Scale-Up project, national screening guidelines and WHO recommendations. We optimistically assumed $70 \%$ of women had access to routine screening and attended an initial visit at a screening facility (ie, a primary healthcare facility). Women could then return to receive screening results and recommendations for any necessary follow-up care. Follow-up could include colposcopy, cryotherapy or triage testing at a referral (ie, higher level) facility, with the exception of Pap triage testing, which was assumed to take place at the screening clinic. At each encounter after the initial screening visit, we assumed $85 \%$ of women complied with each subsequent visit to a screening facility, while $40 \%$ complied with each subsequent visit to a referral facility, consistent with data from Phase 2 of the Scale-Up project. The minimum number of visits required for treatment in a single screening episode was four for Pap, three for HPV-Cryo, three for HPV-VIA and five for HPV-Pap. In the HPV-VIA and HPV-Pap strategies, women who were HPV positive but negative on the selected triage test were referred to repeat HPV testing in 1 year. In the HPV-Cryo and HPV-VIA strategies, women who were not eligible for treatment with cryotherapy based on visual assessment were referred to colposcopy with biopsy to rule out cancer; in the absence of cancer, these women were referred to treatment with loop electrosurgical excision procedure (LEEP) or cryotherapy.

Screening and treatment parameters are presented in table 1. ${ }^{614} 17$ 27-32 Screening test performance data were drawn from the START-UP project in Nicaragua to reflect local test characteristics. While the START-UP project did not evaluate VIA and Pap as triage tests, we used the VIA and Pap positivity rates in HPV-positive women, along with published studies of triage test performance, to inform triage test sensitivity and specificity ${ }^{33-38}$; in the base case, we optimistically assumed high sensitivity of triage testing.

For all HPV testing strategies, we assumed $20 \%$ of women received provider collection of cervical specimens and $80 \%$ of women self-collected vaginal specimens, consistent with the proportions in the Scale-Up project to date. We weighted cost and health outcomes for provider and self-collection accordingly when aggregating results for the HPV strategies.

\section{Cost data}

All costs were converted to 2015 US $\$$ using GDP deflators and the official exchange rate. ${ }^{39}$ The direct medical costs of screening, diagnosis and treatment of precancer were drawn from the START-UP study (Masaya department) and the Scale-Up project (Carazo, Chontales and Chinandega departments). Direct medical costs included clinical staff time, clinical supplies, drugs, clinical equipment, laboratory staff time, laboratory supplies and laboratory equipment. Direct non-medical costs included women's round-trip transportation costs to health facilities and were based on estimates provided by Scale-Up project staff to represent average transportation costs in the Carazo, Chontales and Chinandega departments. To account for the opportunity cost of women's time spent travelling to, waiting for or receiving care, we used time estimates from the START-UP and Scale-Up projects and valued women's time using Nicaragua's monthly minimum wage to serve as a proxy for the societal value of women's time. Figure 2 displays the categorical breakdown of undiscounted costs for the screening visits over the course of a woman's screening-eligible years, with Pap versus careHPV testing.

Data on programmatic costs are limited, but for HPV strategies, we included the cost of training sessions for health providers offering HPV-based screening, outreach workers, laboratory technicians and providers offering VIA and cryotherapy. While women who self-collected HPV specimens in the Scale-Up project primarily did so in a community setting, micro-costing data were not available for self-collection performed outside of the clinic, so we assumed clinic-based self-collection. However, we conservatively included the cost of training outreach workers to represent a known programmatic cost as self-collection efforts are shifting to community settings.

Data on the costs of treating cervical cancer were unavailable for Nicaragua, so we estimated direct medical, direct non-medical and patient and support person time costs using data from El Salvador. ${ }^{29}$

Selected cost data are presented in table 1. Further details on cost data are provided in the online supplementary appendix.

\section{Sensitivity analyses}

We performed sensitivity analysis to examine the impact of independently varying uncertain parameters, including Pap test performance, triage test performance in HPV-positive women, colposcopy performance, screening coverage, visit compliance, eligibility for cryotherapy following a positive screening and triage test, treatment effectiveness, discount rate and cost data. Ranges selected for sensitivity analysis are displayed in table 1 .

\section{Scenario analysis}

The base-case and sensitivity analyses assumed the availability of all strategies (ie, Pap, HPV-Cryo, HPV-VIA and HPV-Pap). Additionally, we performed a scenario analysis in which we assumed HPV-Cryo was not available for logistical and programmatic reasons (ie, limited access to cryotherapy equipment and gas).

\section{RESULTS}

Base case: population-level health benefits and costeffectiveness analysis

HPV-based screening strategies were more effective than Pap testing. Among the HPV strategies, HPV-Cryo (every 5 years) was the most effective strategy; under base-case assumptions, it reduced the lifetime risk of cervical cancer by $29.5 \%$ on average (range, 25.2\%-33.6\%). HPV-VIA (every 5 years) reduced cancer risk by $19.4 \%$ (range, 16.2\%-22.6\%), while HPV-Pap reduced cancer 


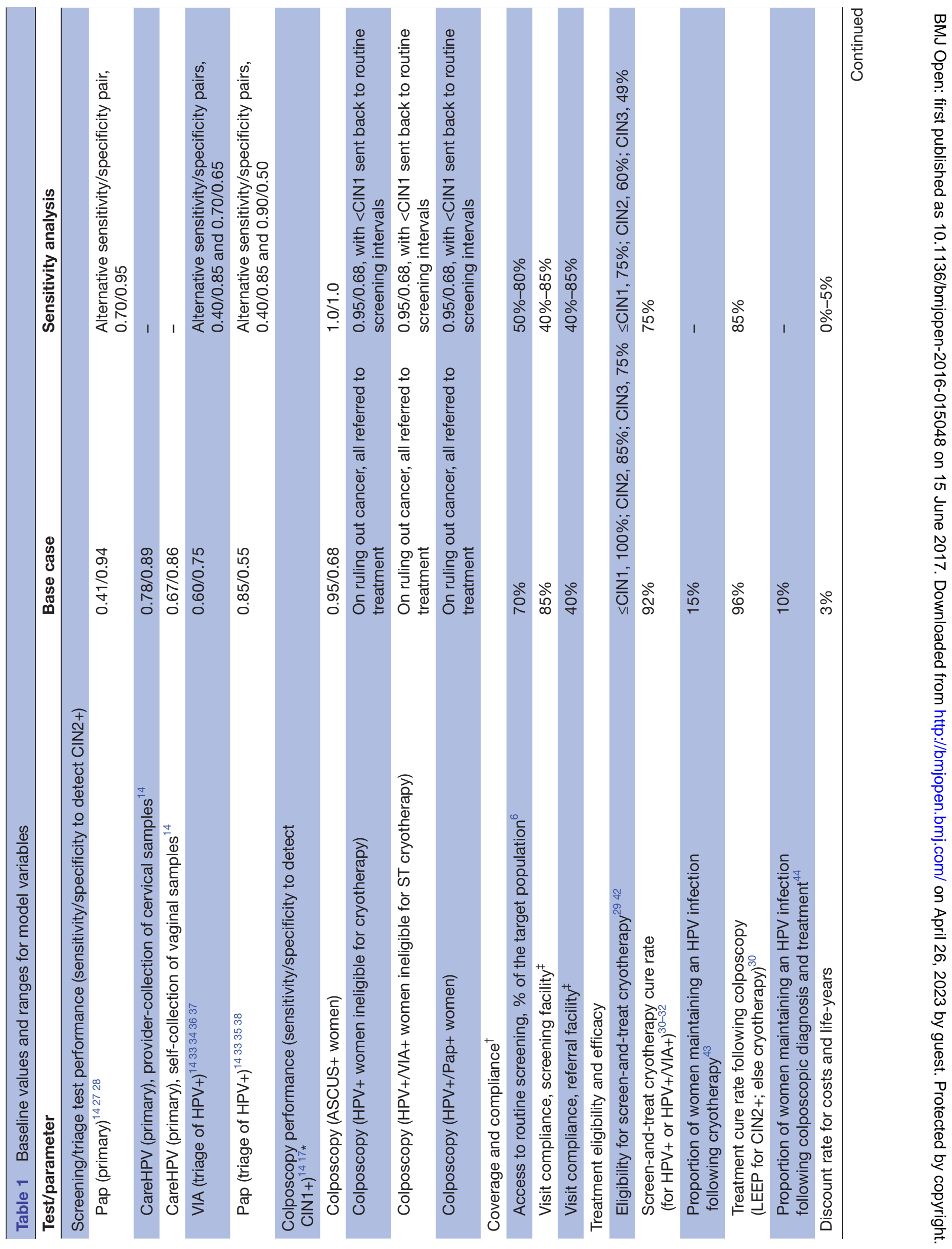




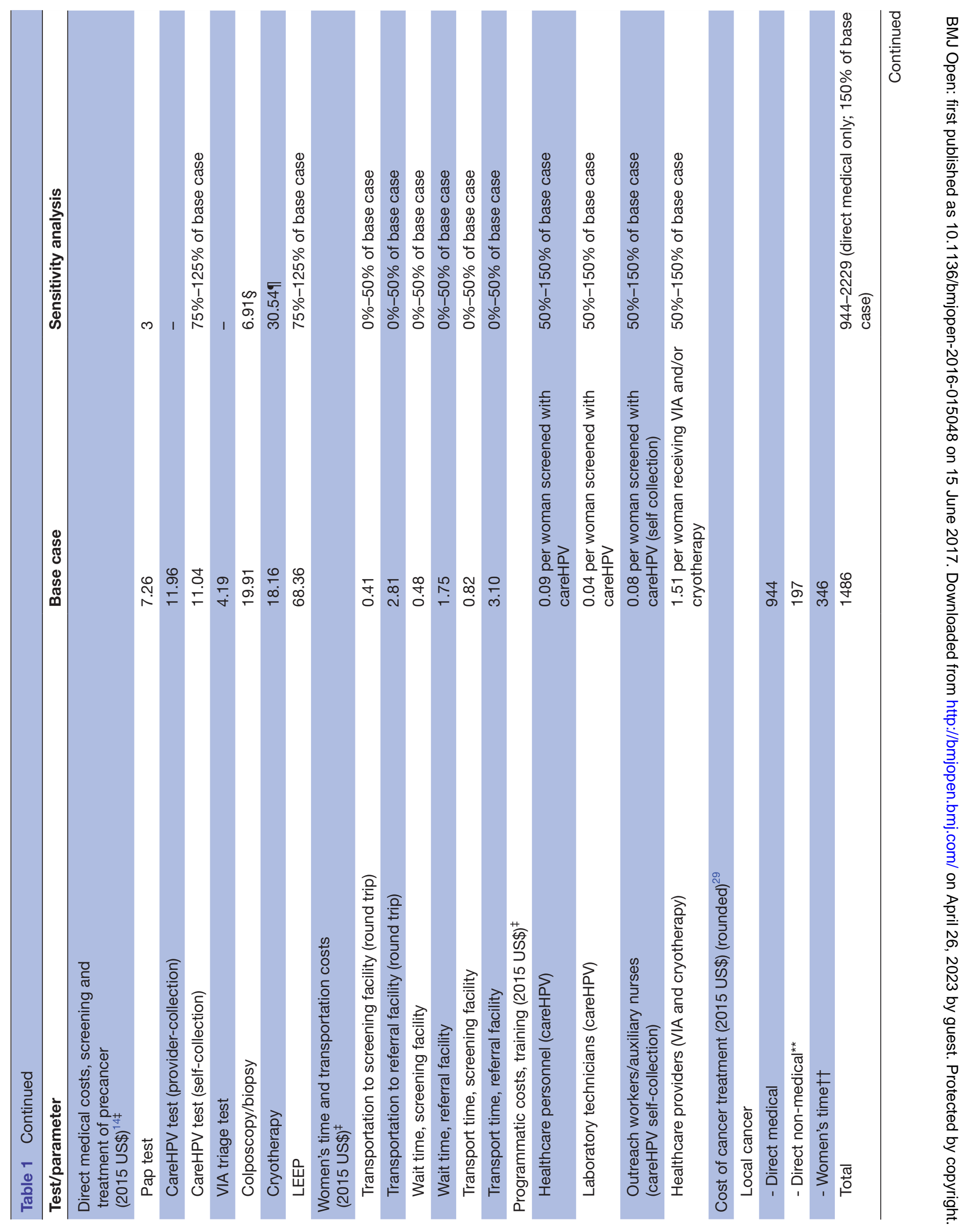




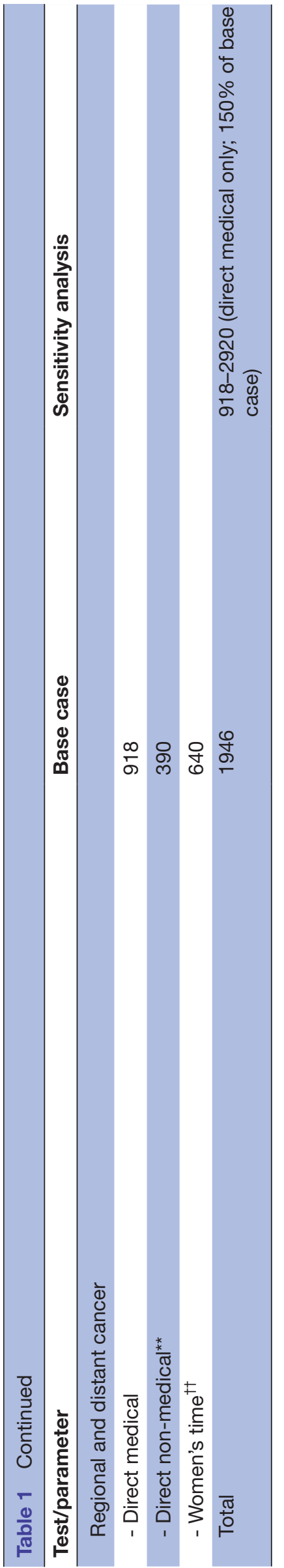

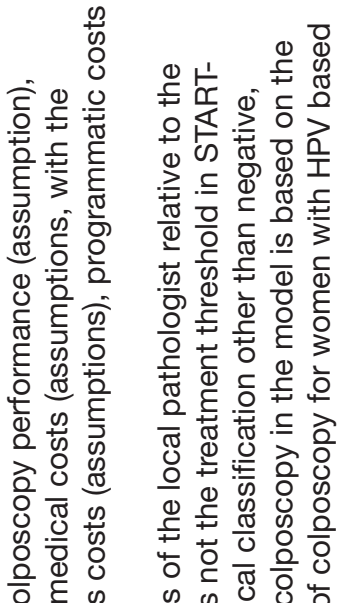

㐫

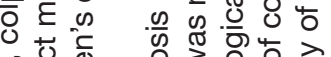

बิ

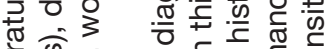

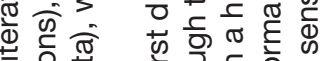

要

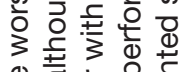

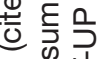

.

ब䒕市这

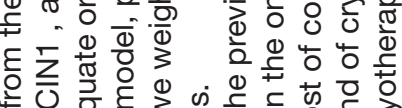

क⿺

으 힝

$\stackrel{\oplus}{\rightleftharpoons}$

옹

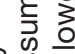

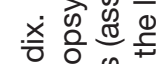

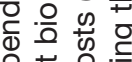

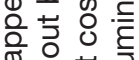

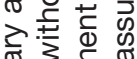

要

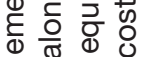

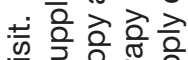

$>$ के

की 4 \&

을 응 응 충

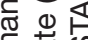

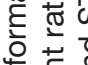

⿺辶大

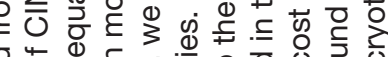

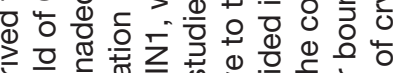

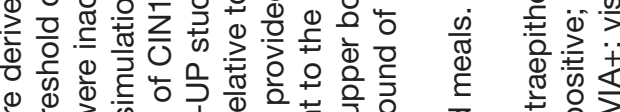

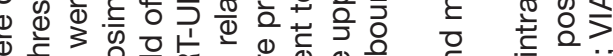

कु

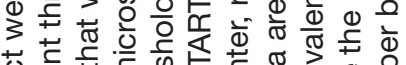

峞芯

要茪

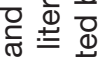

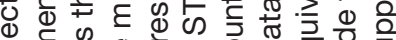

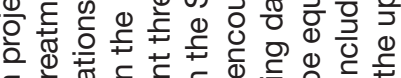

융

为

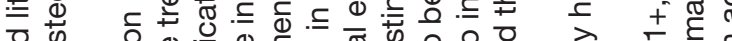

की

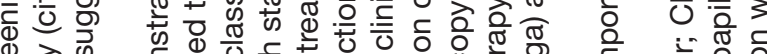

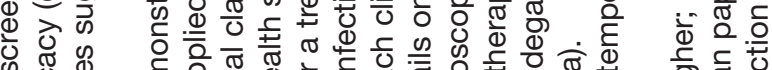

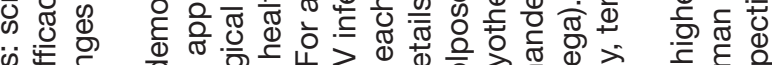

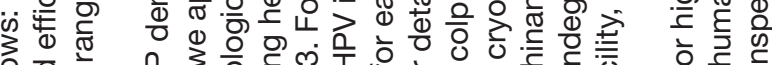

兰航

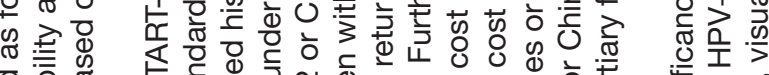

ס

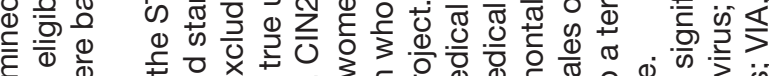

हो

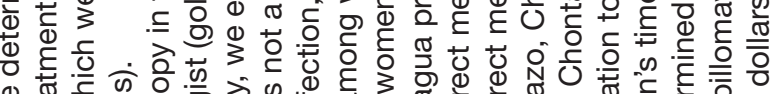

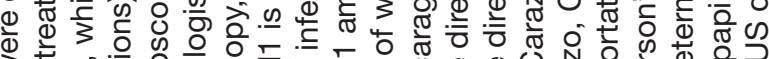

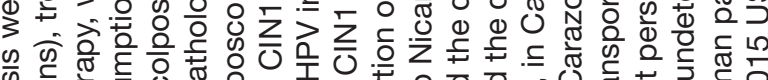
N 을

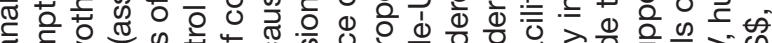
ब

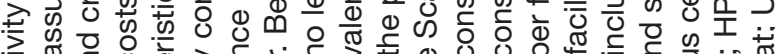

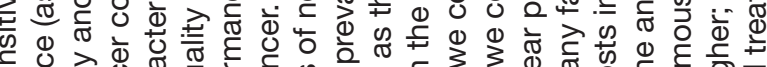
d tᄒ क ब क व 屯。

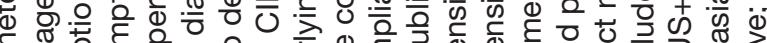

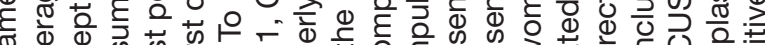
ॠ 


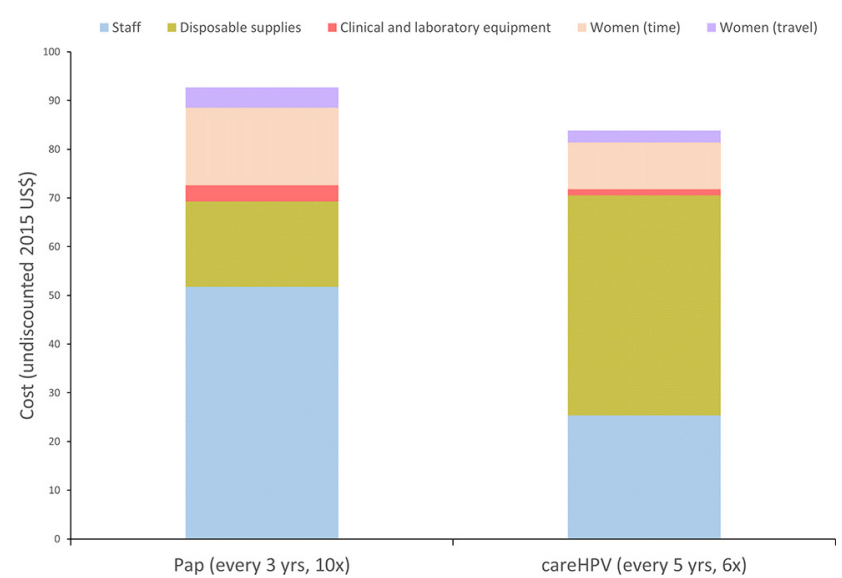

Figure 2 Cervical cancer screening cost per woman over the duration of screening eligibility, by cost component: Pap testing (every 3 years) versus careHPV testing (every 5 years). Bars indicate the undiscounted cost (2015 US\$) of screening with Pap testing (offered 10 times between ages 30 and 59 years) versus careHPV testing (offered six times between ages 30 and 59 years), by cost component. Only screening costs are shown; costs associated with recommended management following a positive screening test are not included. $6 \times$, delivered six times over the course of screening eligible ages 30 to $59 ; 10 \times$, delivered 10 times over the course of screening eligible ages.

risk by $12.2 \%$ (range, $10.2 \%-14.5 \%$ ). Pap (every 3 years) reduced cancer risk by $10.8 \%$ (range, $8.7 \%-13.4 \%$ ). Under base-case assumptions, HPV-Cryo was both less costly and more effective than all other strategies, thus dominating HPV-VIA, HPV-Pap and Pap alone. With an ICER of US $\$ 320$ per year of life saved (YLS), HPV-Cryo every 5 years would be considered 'very cost-effective' given Nicaragua's per capita GDP of US\$2090. The total discounted lifetime cost per woman and life expectancy associated with each screening strategy is presented in figure 3 .

\section{Sensitivity analysis}

While HPV-Cryo remained the most effective strategy across all sensitivity analyses, the magnitude of reduction in lifetime risk of cancer was dependent on screening coverage of the target population and compliance with recommended follow-up. When coverage was $50 \%$ and all other parameters were held constant at base-case values, HPV-Cryo reduced cancer risk by an average of 21.1\%; HPV-VIA, HPV-Pap and Pap yielded average cancer risk reductions of $13.9 \%, 8.7 \%$ and $7.7 \%$, respectively. As coverage increased to $80 \%$, all else being equal, HPV-Cryo reduced cancer risk by an average of $33.5 \%$, while HPV-VIA, HPV-Pap and Pap yielded average cancer risk reductions of $22.1 \%, 14.0 \%$ and $12.4 \%$, respectively (see online supplementary appendix). Figure 4 displays the impact of visit compliance on lifetime risk of cancer. When compliance with visits to all facilities (ie, for both screening and referral) was low at 40\%, HPV-Cryo remained the most effective strategy but only reduced cancer risk by $16.2 \%$; Pap had little health impact at this

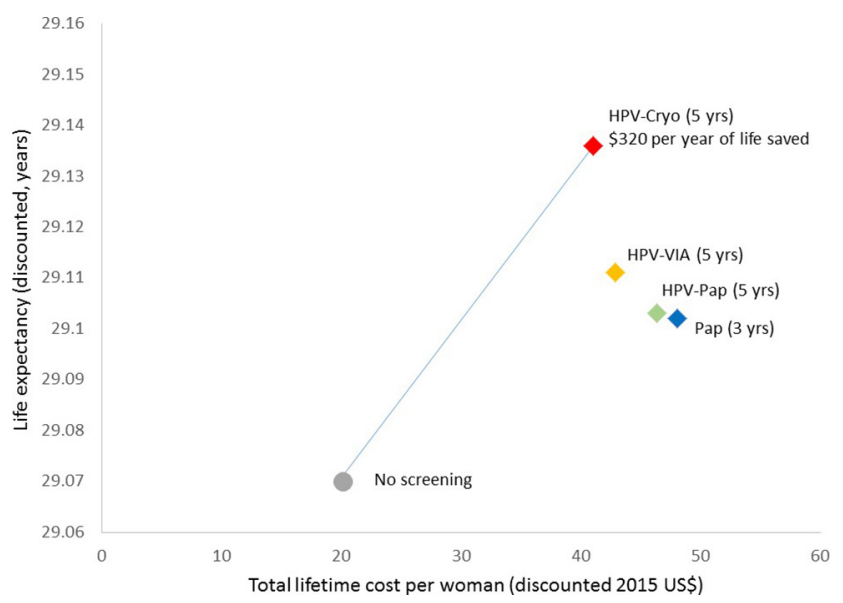

Figure 3 Cost-effectiveness analysis: base-case results. The graph displays the discounted lifetime costs ( $x$ axis; in 2015 US\$) and life expectancy (y axis) associated with each screening strategy (Pap testing every 3 years, careHPV every 5 years with cryotherapy for HPV-positive eligible women (HPV-Cryo), careHPV every 5 years with visual inspection with acetic acid (VIA) triage of HPV-positive women (HPV-VIA) and careHPV every 5 years with Pap triage of HPV-positive women (HPV-Pap)), under base-case assumptions. The costeffectiveness associated with a change from one strategy to a more costly alternative is represented by the difference in cost divided by the difference in life expectancy associated with the two strategies. The curve indicates the strategies that are efficient because they are more effective and either (1) cost less or (2) have a more attractive cost-effectiveness ratio than less effective options. The incremental costeffectiveness ratio (ICER) is the reciprocal of the slope of the line connecting the two strategies under comparison. In the base-case analysis, HPV-Cryo every 5 years was less costly and more effective than other screening strategies considered and was thus a dominant strategy with an ICER of US $\$ 320$ per year of life saved. HPV-Cryo, HPV testing with cryotherapy for HPV-positive women; HPV-Pap, HPV testing with Pap triage of HPV-positive women; HPV-VIA, HPV testing with visual inspection with acetic acid triage of HPVpositive women; yrs, years.

level of compliance, reducing cancer risk by only $5.4 \%$. As compliance at all facilities rose to $85 \%$, HPV-Cryo reduced cancer risk by $47.9 \%$; HPV-VIA, HPV-Pap and Pap reduced cancer risk by $42.0 \%, 40.7 \%$ and $35.7 \%$ respectively.

In addition to remaining the most effective strategy across all sensitivity analyses, HPV-Cryo remained the most efficient strategy as well. HPV-Cryo remained the least costly and most effective strategy with a stable ICER of US\$320 per YLS when (1) Pap test performance (as a primary screening test) improved, (2) VIA and Pap triage test performance improved, (3) colposcopy was assumed to be perfect and (4) the direct medical cost of LEEP was varied from $75 \%$ to $125 \%$ of the base case. Despite slight fluctuation in the ICER, HPV-Cryo also remained the least costly and most effective strategy as (1) screening coverage varied from $50 \%$ to $80 \%$; (2) visit compliance varied from $40 \%$ to $85 \%$ per visit; (3) the screen-and-treat cryotherapy cure rate was reduced to 


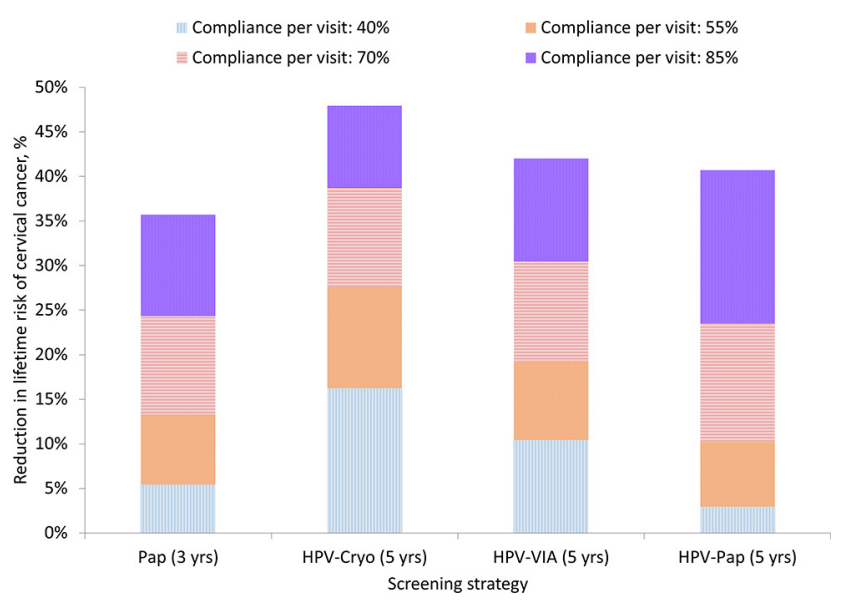

Figure 4 Reduction in lifetime risk of cervical cancer, by compliance level. Bars indicate the per cent reduction in lifetime risk of cervical cancer for each screening strategy (Pap testing every 3 years, careHPV every 5 years with cryotherapy for HPV-positive women (HPV-Cryo), careHPV every 5 years with visual inspection with acetic acid (VIA) triage of HPV-positive women (HPV-VIA) and careHPV every 5 years with Pap triage of HPV-positive women (HPV-Pap)) as compliance per visit within a screening episode increases. Compliance is defined as the proportion of women who return for each clinical encounter, relative to the previous visit. Coverage of the target population is assumed to be $70 \%$. While the base-case analysis assumed $85 \%$ compliance for visits at screening facilities and $40 \%$ compliance for visits at referral facilities (for diagnosis and treatment), the graph displays cancer risk reduction assuming the specified compliance level at all visits, regardless of facility type.

$75 \%$; (4) eligibility for cryotherapy was reduced to $75 \%$ or less; (5) the treatment cure rate following colposcopy was reduced to $85 \%$; (6) women receiving treatment following colposcopy in the Pap and HPV-Pap strategies were assumed to receive cryotherapy instead of the more costly LEEP; (7) the discount rate was varied from $0 \%$ to $5 \%$; $(8)$ only payer costs were considered, excluding women's time and transportation costs; (9) the direct medical cost of HPV self-collection was varied from $75 \%$ to $125 \%$ of the base case; (10) the direct medical cost of cryotherapy was increased to $170 \%$ of the base case; (11) the direct medical cost of colposcopy was reduced to $35 \%$ of the base case; (12) programmatic costs associated with HPV-based screening were varied from $50 \%$ to $150 \%$ of the base case; (13) women's time and transportation costs were reduced to $50 \%$ of the base case; and (14) the costs of cancer treatment ranged from including only direct medical costs to $150 \%$ of the base case (figure 5). Among variables considered, compliance per visit appears to have the greatest impact on the ICER for HPV-Cryo, with $40 \%$ compliance yielding an ICER of US $\$ 580$ per YLS and $85 \%$ compliance yielding an ICER of US $\$ 190$ per YLS. Even when visit compliance is low, HPV-Cryo would be considered very cost-effective.

When only payer costs were considered (ie, women's time and transportation costs were excluded), the total lifetime cost per woman was lower for all strategies and Pap every 3 years was slightly less costly (although still slightly less effective) than HPV-Pap every 5 years. HPV-Cryo remained the most effective and efficient strategy, with an ICER of US\$270 per YLS (see online supplementary appendix).

The only scenario in which Pap testing every 3 years was the least costly strategy occurred when the direct medical cost of Pap testing was US $\$ 3$ (base case, US $\$ 7.26$ ), a value commonly cited for the cost of Pap in Nicaragua, though the source of this estimate is unknown. However, Pap remained the least effective strategy, and HPV-Cryo had a lower cost-effectiveness ratio, maintaining an ICER of US $\$ 320$ per YLS (see online supplementary appendix).

\section{Scenario analysis: HPV-Cryo unavailable}

When we assumed HPV-Cryo was not available as a screening strategy, HPV-VIA was the least costly and most effective strategy in the base-case and most sensitivity analyses, with a base-case ICER of US $\$ 550$ per YLS (see online supplementary appendix). Exceptions included the following circumstances: (1) when the direct medical cost of Pap was US\$3, Pap alone had a more attractive ICER (US\$530), although was less effective than HPV-VIA (US\$630 per YLS); (2) Pap performance in the general screening population was improved and Pap alone became the most effective strategy, with an ICER of US\$540 per YLS; (3) VIA test sensitivity in HPV-positive women was only 0.40 , in which case HPV-VIA had an ICER of US $\$ 726$ per YLS, but HPV-Pap was more effective with an ICER of US\$3260.

\section{DISCUSSION}

Using implementation data from the Scale-Up projectwhich aims to facilitate institutionalisation of HPV testing at the national level in Guatemala, Honduras and Nicaragua-we estimated the long-term health impact and value of careHPV testing in Nicaragua's public health system. We found that screening algorithms consisting of HPV testing at 5-year intervals would be less costly and more effective than screening with Pap testing at 3-year intervals. Furthermore, HPV testing followed by treatment with cryotherapy for all eligible HPV-positive women would be less costly and more effective than HPV testing followed by triage testing with either VIA or Pap for HPV-positive women. A screen-and-treat HPV programme would be a very cost-effective intervention in Nicaragua, with an ICER of US\$320 per YLS under base-case assumptions. These findings were robust across sensitivity analyses. The comparatively large health benefits and efficiency of HPV-Cryo can largely be attributed to the relatively low number of visits to healthcare facilities and the high sensitivity of the careHPV test to detect both CIN2 and oncogenic HPV infections with the potential to develop into precancer.

We found that screening coverage of the target population had a considerable impact on achievable reductions in cervical cancer risk, with HPV-Cryo yielding the 


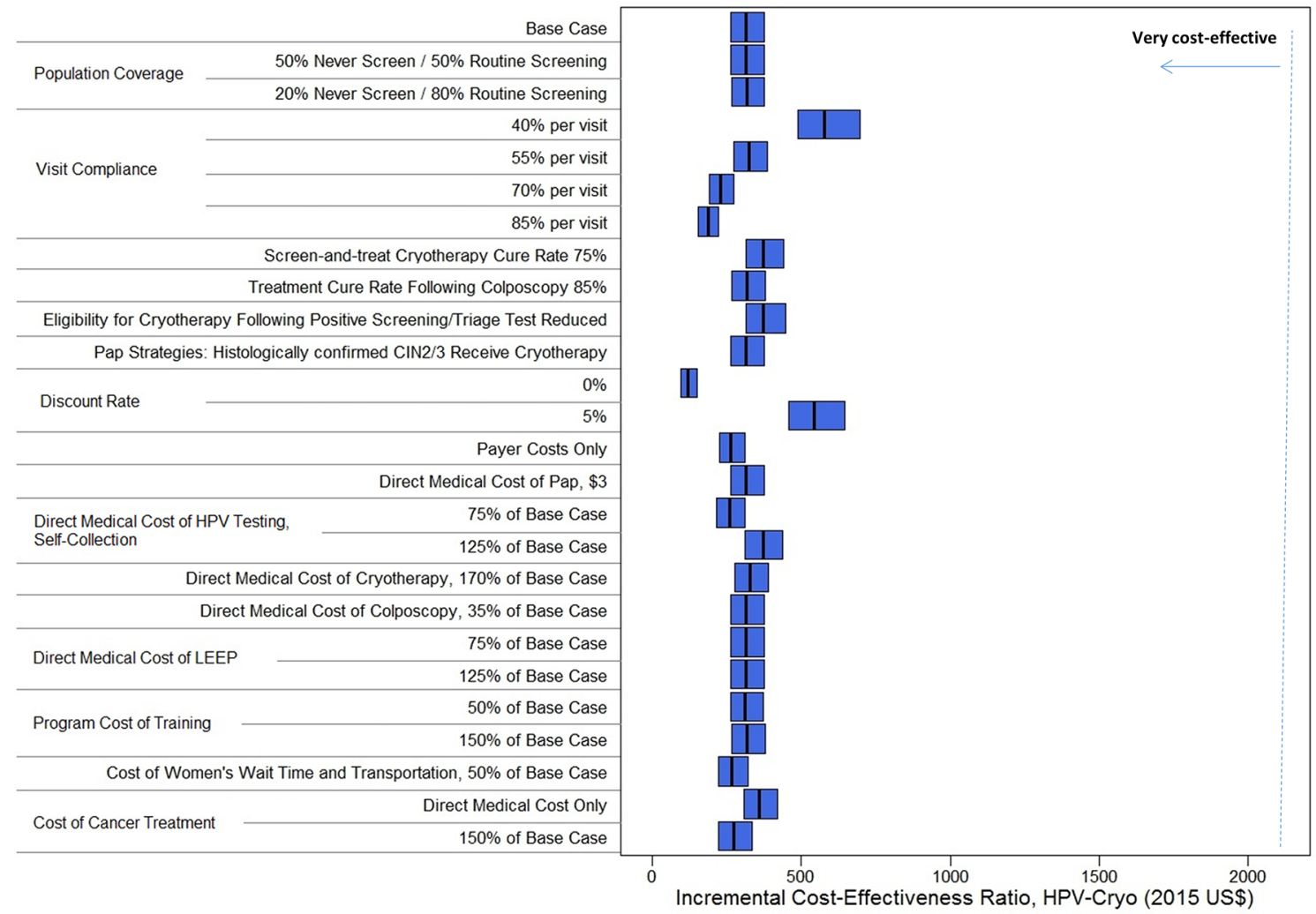

Figure 5 Base-case and sensitivity analyses: incremental cost-effectiveness ratios, HPV cryotherapy (HPV-Cryo) strategy. Incremental cost-effectiveness ratios (ICER) are presented (x-axis, 2015 US\$ per year of life saved) for the base-case and sensitivity analyses (y-axis). The blue bars represent the range of the ICER for HPV-Cryo every 5 years across the 50 input parameter sets, with the ICER of the mean costs divided by the mean effects demarcated by a black line. The dashed blue line indicates Nicaragua's per capita gross domestic product (GDP), at US\$2,090, assuming this is the threshold that designates interventions as 'very cost-effective'.

greatest risk reduction. Due to proportional increases in both costs and health benefits, the ICER for HPV-Cryo remained stable as coverage increased from $50 \%$ to $80 \%$. Compliance with recommended follow-up was a key driver of both achievable reductions in cancer risk and the ICER of HPV-Cryo. As the proportion of women who returned for each clinical encounter (relative to the previous visit) increased from $40 \%$ to $85 \%$, the effectiveness or cancer benefit associated with HPV-Cryo rose from $16.2 \%$ to $47.9 \%$ as more women were linked to treatment; the ICER fell from US $\$ 580$ per YLS to US $\$ 190$ per YLS as more cancers were averted. Thus, improved efforts to successfully navigate women to recommended follow-up will enhance screening programme effectiveness and efficiency.

While substantially reducing the cost of Pap testing to US\$3 (less than half of the base case value) made Pap the strategy with the lowest per-woman lifetime costs, the confluence of low test sensitivity and the high number of health facility visits needed to complete screening, diagnostic follow-up and treatment made Pap the least effective strategy since women are lost to follow-up with each additional required visit. Even in this low-cost Pap scenario, HPV-Cryo remained the most- effective and cost-effective strategy.
When we assumed a scenario in which HPV-Cryo was not available for logistic and programming reasons, we found that HPV-VIA was usually the least costly and most effective of the remaining strategies. Although the ICER was less attractive than the ICER associated with HPV-Cryo in the main analysis, it was below Nicaragua's per capita GDP.

There are several limitations to this analysis. We did not consider alternative screening intervals or ages for each strategy, but rather restricted the analysis to the ages and intervals currently under consideration by the Nicaraguan Ministry of Health. It is likely that an increased screening interval and fewer lifetime screens will also be cost-effective, although health benefits may be reduced; we demonstrated in a previous analysis that screening once or three times in a woman's lifetime with careHPV would be very cost-effective in Nicaragua. ${ }^{17}$ The screening algorithms, as modelled, reflect the prototypical structure of a screening episode and the type of facility at which visits usually take place, but do not capture variation due to geography or health facility capacity. Furthermore, the modelled screening algorithms do not entirely reflect the complex downstream follow-up of screen-positive women that is embodied in the Ministry of Health's screening guidelines. In 
simplifying the downstream follow-up for modelled strategies, we may have underestimated the costs and overestimated the benefits relative to the national guidelines, which call for additional follow-up prior to treatment. However, the modelled strategies would likely bias the analysis in favour of Pap and HPV triage strategies. We also did not consider a strategy in which women with ASCUS were referred directly to cryotherapy instead of colposcopy because that approach is not recommended by the WHO guidelines or by any professional medical society to the best of our knowledge. We did not consider quality of life impact nor the potential disutilities or harms that might be associated with overtreatment.

While we adopted a micro-costing approach to leverage data from the START-UP and Scale-Up projects in Nicaragua, there remain limitations to our cost estimates. First, individual-level data for each woman were not available; thus, our estimates represent average costs in the project populations. Second, we did not have information on the costs associated with HPV self-collection in community settings, where most self-collection takes place. Instead, we assumed all self-collection took place at the clinic. Compared with clinic-based efforts, community-based self-collection may be associated with lower costs for women's time and travel, and higher direct medical and programmatic costs due to outreach worker involvement in facilitating screening and delivering results. Third, our estimates of programmatic costs were restricted to training sessions, and we did not have information on the costs of social mobilisation and outreach, patient navigation and support or infrastructural improvements that would be required to successfully scale up a screening programme. Fourth, we valued women's time based on the minimum wage in Nicaragua. This may be a conservative estimate if most women attending screening are formally employed; conversely, it may overestimate the societal value of women's time spent working in the informal sector or at home. Finally, we extrapolated the cost of cancer treatment using data from El Salvador. ${ }^{29}$ Despite these limitations, extensive sensitivity analyses on cost components indicate that HPV-Cryo is robustly the most efficient strategy.

As implementation of HPV testing continues, particularly without triage testing, the health system's capacity to provide cryotherapy will likely need to increase. While the use of triage testing (either with Pap or HPV) reduces the number of cryotherapy procedures performed, we found that the lower sensitivity of triage testing (resulting in more false negatives) led to a decline in health benefits as fewer women with persistent HPV infection and precancer received treatment. The cost savings associated with fewer cryotherapy procedures were outweighed by increased costs of additional follow-up and cancer treatment in triage-negative women. A sensitivity analysis on the cost of cryotherapy revealed that HPV-Cryo remained the dominant strategy even when costs increased to $170 \%$ of the base case. However, we did not explicitly consider the costs of increasing access to cryotherapy machines or the implications of gas stock-outs, which have been identified as barriers in some low-income and middle-income countries. ${ }^{40}$ New ablative technologies currently undergoing testing are smaller, portable and do not require gas. Thermal-coagulation has been used in the UK for more than 30 years, and now it is being used in several low-income and middle-income countries, including as part of a 'screen-and-treat' programme in Malawi, ${ }^{41}$ and is currently undergoing testing in Latin America. If newer technologies demonstrate cure rates similar to cryotherapy, the cost-effectiveness of screen-and-treat algorithms may improve along with access to treatment.

In summary, using data from the Scale-Up implementation project in Nicaragua, we found that HPV testing followed by cryotherapy for eligible HPV-positive women (a screen-and-treat approach) was a very cost-effective intervention in Nicaragua. As the HPV-Cryo algorithm was not implemented in phase 2 of the Scale-Up project, compliance and cost estimates may need to be further honed to reflect improvements in capacity for cryotherapy if HPV-Cryo is implemented going forward. While it is too early to assume that costs and health impact from phase 2 of implementation are generalisable to other departments in Nicaragua or other settings in Central America, extensive sensitivity analyses indicate the robustness of findings. An HPV-based screening algorithm involving a similar screen-and-treat approach was recently found to be a good value for public health dollars in El Salvador, ${ }^{29}$ where a national scale up is underway. It is important to note that a favourable cost-effectiveness profile does not guarantee that HPV-Cryo will be affordable or feasible in a lower-middle-income country like Nicaragua. Both the cost-effectiveness ratio, budgetary impact and health system infrastructure need to be favourable for screening programs to be sustainable. We present these findings to inform evidence-based decision making around national screening guidelines, programme design and implementation and budgeting for infrastructural improvements and procurement of HPV tests in Nicaragua.

Acknowledgements We gratefully acknowledge the efforts of Movicáncer of Nicaragua in gathering costing data.

Contributors All authors developed the analysis plan and interpreted the data. NGC, MM, JJ, FH and JJK conceptualised the study. MM, JJ, FH and EV collected implementation data. NGC conducted data analysis and wrote the first draft of the report and revised subsequent drafts. JJ was the principal investigator of the START-UP and Scale-Up projects. JJK was the principal investigator overseeing microsimulation model development. All authors contributed to and approved the final report.

Funding This work was supported by the Bill \& Melinda Gates Foundation.

Disclaimer The findings and conclusions contained within are those of the authors and do not necessarily reflect positions or policies of the Bill \& Melinda Gates Foundation. The funders had no role in study design; data collection, analysis and interpretation; preparation of the manuscript; or decision to submit the article for publication. 
Competing interests $\mathrm{JJ}$ was the director of the START-UP demonstration projects and received all tests used in the study as a donation from Qiagen; no other relationships or activities that could appear to have influenced the submitted work. JJ was the co-owner and Deputy Manager of Onco Prev International, a Peruvian company, from 2012 through March 2017. Onco Prev International offers cervical cancer screening services and in 2016 also began positioning for distribution of medical devices including colposcopes and the Liger thermocoagulator. Onco Prev International did not commercialize any medical instrument during the time $\mathrm{JJ}$ was part of the company.

Provenance and peer review Not commissioned; externally peer reviewed.

Data sharing statement PATH provides technical assistance to the government of Nicaragua and has access to screening indicators through partnership with Movicáncer, a local non-governmental organisation partner in Nicaragua. Movicáncer designed the health information system used by the Ministry of Health to track screening and treatment of women's cancers. The Ministry of Health protects individually identifiable information; PATH received de-identified and consolidated data on visit compliance from Movicáncer. Costing data were collected by Movicáncer through consultation with Ministry of Health personnel, mostly via phone interviews. No individual identifiers were collected. These data are not available from any public source. A supplementary appendix has been provided to describe costing data and the microsimulation model in detail.

Open Access This is an Open Access article distributed in accordance with the terms of the Creative Commons Attribution (CC BY 4.0) license, which permits others to distribute, remix, adapt and build upon this work, for commercial use, provided the original work is properly cited. See: http://creativecommons.org/ licenses/by/4.0/

(c) Article author(s) (or their employer(s) unless otherwise stated in the text of the article) 2017. All rights reserved. No commercial use is permitted unless otherwise expressly granted.

\section{REFERENCES}

1. Ferlay J, Soerjomataram I, Ervik M, et al. GLOBOCAN 2012 v1.0, Cancer Incidence and Mortality Worldwide: IARC CancerBase No. 11 [Internet]. Lyon, France: International Agency for Research on Cancer, 2013.

2. Walboomers JM, Jacobs MV, Manos MM, et al. Human papillomavirus is a necessary cause of invasive cervical cancer worldwide. J Pathol 1999;189:12-19.

3. Rodríguez AC, Schiffman M, Herrero R, et al. Longitudinal study of human papillomavirus persistence and cervical intraepithelia neoplasia grade 2/3: critical role of duration of infection. J Natl Cancer Inst 2010;102:315-24.

4. Kitchener HC, Castle PE, Cox JT. Chapter 7: Achievements and limitations of cervical cytology screening. Vaccine 2006;24 Suppl 3:S63-S70.

5. Denny L, Quinn M, Sankaranarayanan R. Chapter 8: screening for cervical cancer in developing countries. Vaccine 2006;24 Suppl 3:S71-S77.

6. Institut Catala d'Oncologia. HPV Information Centre. 2014 www. hpvcentre.net.

7. Vastbinder MB, Castillo C, Bekkers RL. Barriers to follow-up of abnormal Papanicolaou smears among women in Leon, Nicaragua. Trop Doct 2010;40:22-6.

8. Khan MJ, Castle PE, Lorincz AT, et al. The elevated 10-year risk of cervical precancer and cancer in women with human papillomavirus (HPV) type 16 or 18 and the possible utility of type-specific HPV testing in clinical practice. J Natl Cancer Inst 2005;97:1072-9.

9. World Health Organization. In WHO Guidelines for Screening and Treatment of Precancerous Lesions for Cervical Cancer Prevention. Geneva: World Health Organization, 2013.

10. Arrossi S, Thouyaret L, Herrero R, et al. Effect of self-collection of HPV DNA offered by community health workers at home visits on uptake of screening for cervical Cancer (the EMA study): a population-based cluster-randomised trial. Lancet Glob Health 2015;3:e85-e94.

11. Moses E, Pedersen HN, Mitchell SM, et al. Uptake of communitybased, self-collected HPV testing vs. visual inspection with acetic acid for cervical cancer screening in Kampala, Uganda: preliminary results of a randomised controlled trial. Trop Med Int Health 2015;20:1355-67.

12. Lazcano-Ponce E, Lorincz AT, Cruz-Valdez A, et al. Self-collection of vaginal specimens for human papillomavirus testing in cervical cancer prevention (MARCH): a community-based randomised controlled trial. Lancet 2011;378:1868-73.

13. Racey CS, Withrow DR, Gesink D. Self-collected HPV testing improves participation in cervical cancer screening: a systematic review and meta-analysis. Can J Public Health 2013;104:e159-66.

14. Jeronimo J, Bansil P, Lim J, et al. A multicountry evaluation of careHPV testing, visual inspection with acetic acid, and Papanicolaou testing for the detection of cervical Cancer. Int $J$ Gynecol Cancer 2014;24:576-85.

15. Qiao YL, Sellors JW, Eder PS, et al. A new HPV-DNA test for cervical-cancer screening in developing regions: a cross-sectional study of clinical accuracy in rural China. Lancet Oncol 2008;9:929-36.

16. Jeronimo J, Holme F, Slavkovsky R, et al. Implementation of HPV testing in Latin America. J Clin Virol 2016;76 (Suppl 1):S69-S73.

17. Campos NG, Tsu V, Jeronimo J, et al. When and how often to screen for cervical cancer in three low- and middle-income countries: a cost-effectiveness analysis. Papillomavirus Res 2015;1:38-58.

18. Campos NG, Burger EA, Sy S, et al. An updated natural history model of cervical cancer: derivation of model parameters. Am J Epidemiol 2014;180:545-55.

19. World Health Organization. Macroeconomics and health: investing in health for economic development: report of the Commission on Macroeconomics and Health. Geneva: World Health Organization, 2001.

20. Tan-Torres Edejer T, Baltussen R, Adam T, Hutubessy R, Acharya A, Evans DB, Murray CJL, et al. eds. Making choices in health: WHO guide to cost-effectiveness analysis. Geneva, Switzerland: World Health Organization, 2003.

21. Jamison DT, Breman JG, Measham AR, Alleyne G, Claeson M, Evans DB, Jha P, Mills A, Musgrove P, et al. eds. Disease control priorities in developing countries. Second edition. Washington, D.C: Oxford University Press and The World Bank, 2006.

22. Muñoz N, Méndez F, Posso H, et al. Incidence, duration, and determinants of cervical human papillomavirus infection in a cohort of colombian women with normal cytological results. J Infect Dis 2004;190:2077-87.

23. Herrero R, Hildesheim A, Rodríguez AC, et al. Rationale and design of a community-based double-blind randomized clinical trial of an HPV 16 and 18 vaccine in Guanacaste, Costa Rica. Vaccine 2008;26:4795-808.

24. Surveillance, Epidemiology, and End Results (SEER) Program. SEER*Stat Database: Incidence - SEER 18 Regs Research Data + Hurricane Katrina Impacted Louisiana Cases Nov 2011 Sub (19732009 varying) - Linked to County Attributes -Total U.S., 1969-2010 Counties. Bethesda, MD: National Cancer Institute, Division of Cancer Control and Population Sciences, Surveillance Research Program, Surveillance Systems Branch, 2012.

25. Sankaranarayanan $\mathrm{R}$, Swaminathan $\mathrm{R}$, Brenner $\mathrm{H}$, et al. Cancer survival in Africa, Asia, and Central America: a population-based study. Lancet Oncol 2010;11:165-73.

26. Stinnett AA, Paltiel AD. Estimating CE ratios under second-order uncertainty: the mean ratio versus the ratio of means. Med Decis Making 1997;17:483-9.

27. Mayrand $\mathrm{MH}$, Duarte-Franco E, Rodrigues I, et al. Human papillomavirus DNA versus Papanicolaou screening tests for cervical Cancer. N Engl J Med 2007;357:1579-88.

28. Ronco G, Dillner J, Elfström KM, et al. Efficacy of HPV-based screening for prevention of invasive cervical cancer: follow-up of four European randomised controlled trials. Lancet 2014;383:524-32.

29. Campos NG, Maza M, Alfaro K, et al. The comparative and costeffectiveness of HPV-based cervical cancer screening algorithms in El Salvador. Int J Cancer 2015;137:893-902.

30. Chirenje ZM, Rusakaniko S, Kirumbi L, et al. Situation analysis for cervical cancer diagnosis and treatment in east, central and southern african countries. Bull World Health Organ 2001;79:127-32.

31. Cremer M, Bullard K, Maza M, et al. Cytology versus visual inspection with acetic acid among women treated previously with cryotherapy in a low-resource setting. Int J Gynaecol Obstet 2010;111:249-52.

32. Sauvaget C, Muwonge R, Sankaranarayanan R. Meta-analysis of the effectiveness of cryotherapy in the treatment of cervical intraepithelial neoplasia. Int J Gynaecol Obstet 2013;120:218-23.

33. Bigoni J, Gundar M, Tebeu PM, et al. Cervical cancer screening in sub-Saharan Africa: a randomized trial of VIA versus cytology for triage of HPV-positive women. Int J Cancer 2015;137:127-34. 
34. Catarino R, Vassilakos $P$, Scaringella $S$, et al. Smartphone use for cervical cancer screening in low-resource countries: a pilot study conducted in Madagascar. PLoS One 2015;10:e0134309.

35. Pan QJ, Hu SY, Guo HQ, et al. Liquid-based cytology and human papillomavirus testing: a pooled analysis using the data from 13 population-based cervical cancer screening studies from China. Gynecol Oncol 2014;133:172-9.

36. Qiao YL, Jeronimo J, Zhao FH, et al. Lower cost strategies for triage of human papillomavirus DNA-positive women. Int J Cancer 2014;134:2891-901.

37. Tebeu PM, Fokom-Domgue J, Crofts V, et al. Effectiveness of a twostage strategy with HPV testing followed by visual inspection with acetic acid for cervical cancer screening in a low-income setting. Int $J$ Cancer 2015;136:E743-E750.

38. Richardson LA, El-Zein M, Ramanakumar AV, et al. HPV DNA testing with cytology triage in cervical cancer screening: influence of revealing HPV infection status. Cancer Cytopathol 2015;123:745-54.
39. World Bank. World Development Indicators. 2016 http://databank. worldbank.org/data/reports.aspx?source=world-developmentindicators.

40. Msyamboza KP, Phiri T, Sichali W, et al. Cervical cancer screening uptake and challenges in Malawi from 2011 to 2015: retrospective cohort study. BMC Public Health 2016;16:806.

41. Campbell $\mathrm{C}$, Kafwafwa $\mathrm{S}$, Brown $\mathrm{H}$, et al. Use of thermo-coagulation as an alternative treatment modality in a 'screen-and-treat' programme of cervical screening in rural Malawi. Int $J$ Cancer 2016;139:908-15.

42. Gage JC, Rodriguez AC, Schiffman M, et al. Treatability by cryotherapy in a screen-and-treat strategy. J Low Genit Tract Dis 2009:13:174-81.

43. Starks D, Arriba LN, Enerson CL, et al. Mexican cervical cancer screening study II: 6-month and 2-year follow-up of HR-HPV women treated with cryotherapy in a low-resource setting. J Low Genit Tract Dis 2014;18:333-7.

44. McClung EC, Blumenthal PD, Efficacy BPD. Efficacy, safety, acceptability and affordability of cryotherapy: a review of current literature. Minerva Ginecol 2012;64:149-71. 